\title{
Resonant passive-active vibration absorber with integrated force feedback control
}

\author{
Høgsberg, Jan Becker; Brodersen, Mark Laier; Krenk, Steen
}

\section{Published in:}

Smart Materials and Structures

Link to article, DOI:

10.1088/0964-1726/25/4/047001

Publication date:

2016

Document Version

Peer reviewed version

Link back to DTU Orbit

Citation (APA):

Høgsberg, J. B., Brodersen, M. L., \& Krenk, S. (2016). Resonant passive-active vibration absorber with integrated force feedback control. Smart Materials and Structures, 25(4), [047001]. https://doi.org/10.1088/0964$1726 / 25 / 4 / 047001$

\section{General rights}

Copyright and moral rights for the publications made accessible in the public portal are retained by the authors and/or other copyright owners and it is a condition of accessing publications that users recognise and abide by the legal requirements associated with these rights.

- Users may download and print one copy of any publication from the public portal for the purpose of private study or research.

- You may not further distribute the material or use it for any profit-making activity or commercial gain

- You may freely distribute the URL identifying the publication in the public portal 


\title{
RESONANT PASSIVE-ACTIVE VIBRATION ABSORBER WITH INTEGRATED FORCE FEEDBACK CONTROL
}

\author{
Jan Høgsberg, Mark L. Brodersen and Steen Krenk
}

Department of Mechanical Engineering, Technical University of Denmark, Nils Koppels Allé, building 403, DK-2800 Kongens Lyngby, Denmark

\begin{abstract}
A general format of a two-terminal vibration absorber is constructed by placing a passive unit in series with a hybrid unit, composed of an active actuator in parallel with a second passive element. The displacement of the active actuator is controlled by an integrated feedback control with the difference in force between the two passive elements as input. This format allows passive and active contributions to be combined arbitrarily within the hybrid unit, which results in a versatile absorber format with guaranteed closed-loop stability. This is demonstrated for resonant absorbers with inertia realized passively by a mechanical inerter or actively by the integrated force feedback. Accurate calibration formulae are presented for two particular absorber configurations and the performance is subsequently demonstrated with respect to both equal modal damping and effective response reduction.
\end{abstract}

\section{INTRODUCTION}

The dynamic response of slender structures is often dominated by resonance, which is effectively reduced by vibration absorbers that are optimally synchronized with the critical vibration form of the structure. The classic concept is that of the mechanical tuned mass damper $[1,2]$ shown in Fig. 1(b), in which the absorber mass is suspended to the flexible structure via a spring and a viscous damper. Mechanical vibration absorbers with two terminal connections can be realized by combining springs and dampers with so-called inerter elements [3, 4], where the inertance is produced by for example a ball-screw driving a flywheel $[4,5]$. Inerter based vibration absorbers have recently been proposed for vibration suppression of structures in $[6,7]$, while [8] considers the calibration of various types of inerter-based absorbers. In smart structures technology two-terminal resonant absorbers can be obtained by piezoelectric $[9,10]$ or electromagnetic $[11,12,13,14]$ shunt circuit concepts, where [14] demonstrates the equivalence between the electromagnetic transducer and the mechanical inerter element.

Mechanical vibration absorbers may also be modified by active elements to enhance the overall damping performance or reduce the required absorber mass [15]. Furthermore, active resonant control strategies may be formulated in terms of so-called virtual vibration absorbers $[16,17]$, which are used to design feedback control laws by equivalence with desirable mechanical absorber systems, or in terms of filtered acceleration or displacement feedback control [18, 19]. The present paper proposes a two-terminal passive-active vibration absorber with integrated force feedback control. The concept of integrated force feedback control is suitable when an active unit is placed in series with a deformable structural element. It has been effectively employed by Preumont and 
co-workers for active control of piezoelectric struts [20], in tendon control applications [21] and in a base-isolation system [22]. Modified integral force feedback formats have recently been considered for damping of structures by a piezoelectric transducer [23] or a hybrid viscous damper [24].

The present absorber is composed of a passive unit in series with a hybrid unit, represented by an active actuator in parallel with a second passive element. The actuator displacement is controlled by integrated force feedback with the difference in measured absorber force between the two passive units as sensor input. Hereby, the resulting (frequency) properties of the hybrid unit appears as the sum of the individual contributions from the active actuator and passive element, respectively. This implies that stability of the hybrid element guarantees stability of the entire passive-active vibration absorber. A resonant form of the passive-active vibration absorber is introduced by creating an inertia force actively by the integrated force feedback control or passively by an inerter element. Calibration formulae are presented for two specific configurations: An inertia-based absorber equivalent to a tuned mass or inerter damper $[1,2,6]$ and a stiffnessbased absorber similar to that of piezoelectric $R L$ shunt damping $[9,10]$. Numerical examples demonstrate the efficiency of the two resonant absorber formats when compared to the optimally tuned viscous damper.

\section{Structure With ABSORBer}

Figure 1 shows a flexible structure with two different types of supplemental devices: (a) a twoterminal absorber and (b) a mechanical tuned mass damper. In the case of harmonic motion with angular frequency $\omega$ the governing structural equations of motion are

$$
\left(-\omega^{2} \mathbf{M}+\mathbf{K}\right) \mathbf{u}+\mathbf{w} f=\mathbf{f}_{e}
$$

where $\mathbf{u}$ contains the harmonic amplitudes of the structural degrees of freedom, $\mathbf{f}_{e}$ represents the corresponding external load components, $\mathbf{M}$ is the mass matrix and $\mathbf{K}$ is the stiffness matrix. The force $f$ from the supplemental absorber acts on the structure at the location(s) defined by the connectivity vector $\mathbf{w}$. The displacement of the structure at the location of the absorber is
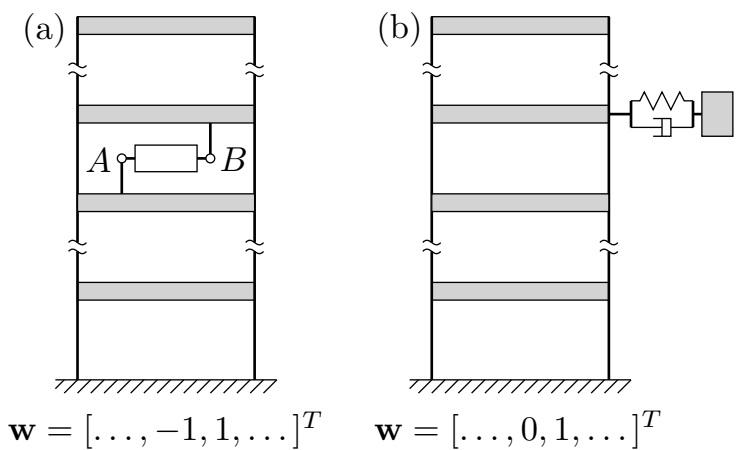

FiguRE 1. Structure with (a) two-terminal vibration absorber and (b) tuned mass damper. 
consequently defined as

$$
u=\mathbf{w}^{T} \mathbf{u}
$$

whereby $f$ produces work through $u$. In Fig. 1(a) the two terminals $A$ and $B$ of the absorber connect two points on the structure with relative displacement $u$, while in Fig. 1(b) $u$ is the displacement of the structure at the location of the tuned mass damper. The connectivity vectors are indicated for both absorber types in Fig. 1.

2.1. Absorber format. A general passive-active vibration absorber format is shown in Fig. 2(a) with terminals $A$ and $B$ representing the attachment points. The absorber is composed of a passive unit with frequency transfer function $H_{d}(\omega)$ in series with a hybrid unit, represented by an active actuator with controllable displacement $q$ and an additional passive element with transfer function $H_{q}(\omega)$ placed in parallel.

The deformation of the passive unit is $u-q$ and the absorber force is therefore given as

$$
f=H_{d}(\omega)(u-q)
$$

In the adjacent hybrid unit the force produced by the additional passive element is

$$
f_{q}=H_{q}(\omega) q
$$

where the actuator displacement $q$ is actively controlled by an integrated force feedback strategy. As shown in Fig. 2(a) the present vibration absorber contains two force sensors, measuring the resulting absorber force $f$ and the force $f_{q}$ in the passive element of the hybrid unit, respectively. The desired absorber format is obtained by an integrated force feedback equation

$$
G_{q}(\omega) q=f-f_{q}
$$

(a)

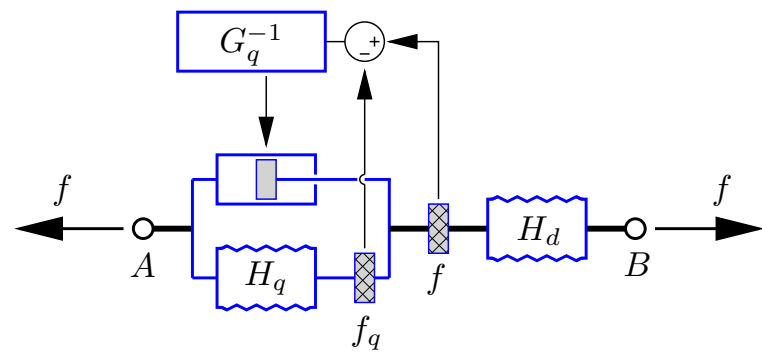

(b)

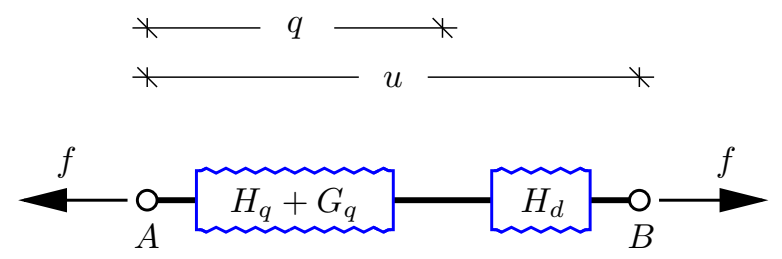

Figure 2. (a) Passive-active vibration absorber with integrated force feedback and (b) equivalent resulting model. 
with the difference in measured force $f-f_{q}$ as control input and with $G_{q}(\omega)$ as integration function. Elimination of $f_{q}$ by substitution of (4) into (5) gives the frequency relation for the hybrid unit,

$$
\left(H_{q}(\omega)+G_{q}(\omega)\right) q=f
$$

Thus, the feedback control in (5), with the force difference $f-f_{q}$ as input, implies that passive components in $H_{q}(\omega)$ and active components in $G_{q}(\omega)$ are combined to form the resulting function $H_{q}(\omega)+G_{q}(\omega)$, operating in series with the passive unit $H_{d}(\omega)$, as illustrated in Fig. 2(b). Furthermore, it follows from (6) that the passive-active vibration absorber has guaranteed stability as long as a mechanical analogy is available for $H_{q}(\omega)+G_{q}(\omega)$, for example composed of springs, dampers and inerters.

The actuator displacement $q$ is obtained as function of the absorber deformation $u$ by substitution of (3) into (5),

$$
q=\frac{H_{d}(\omega)}{H_{d}(\omega)+H_{q}(\omega)+G_{q}(\omega)} u
$$

The passive force $f_{q}$ is then determined by (4), while the absorber force $f$ follows from (6) as

$$
f=\frac{H_{d}(\omega)\left(H_{q}(\omega)+G_{q}(\omega)\right)}{H_{d}(\omega)+H_{q}(\omega)+G_{q}(\omega)} u
$$

It is seen that the denominator is represented by the sum of the three frequency transfer functions, and a resonant form of the vibration absorber therefore requires that at least one of the three transfer functions contain an inertia term proportional to $-\omega^{2}$.

2.2. Resonant absorbers. Numerous damper and absorber configurations are covered by the general format in Fig. 2(a), but in the following only resonant vibration absorbers are considered. Figure 3 shows three versions of the same resonant vibration absorber, with a passive unit

$$
H_{d}(\omega)=i \omega c_{d}+k_{d}
$$

containing a viscous damper $c_{d}$ and spring $k_{d}$ in parallel. In all three cases the resulting transfer function of the hybrid unit is

$$
H_{q}(\omega)+G_{q}(\omega)=-\omega^{2} m_{q}+i \omega c_{q}+k_{q}
$$

with apparent inertance $m_{q}$, viscous parameter $c_{q}$ and stiffness $k_{q}$. In Fig. 3(a) the hybrid unit is realized actively with $H_{q}(\omega)=0$ and $G_{q}(\omega)=-m_{q} \omega^{2}+i \omega c_{q}+k_{q}$, while the entirely passive alternative in Fig. 3(b) is represented by the mechanical elements in $H_{q}(\omega)=-m_{q} \omega^{2}+i \omega c_{q}+k_{q}$ and without force feedback control represented by $G_{q}(\omega) \rightarrow 0$. Because of the equivalence between the two absorber configurations in Figs. 3(a,b) the active hybrid unit in (a) has guaranteed stability. It follows from the additive format in (6), also illustrated in Fig. 2(b), that a passive-active form of the hybrid unit can be obtained by simply combining the passive and active elements in $H_{q}(\omega)$ and $G_{q}(\omega)$ to form the sum $H_{q}(\omega)+G_{q}(\omega)$ in (6). Figure 3(c) shows a passive-active hybrid unit, where the inertia contribution $-\omega^{2} m_{q}$ is introduced by a mechanical inerter with inertance $m_{q}$, while stiffness and damping in this case are realized actively by the first order integration filter $G_{q}(\omega)=i \omega c_{q}+k_{q}$. This combined configuration could be of practical interest because mechanical inerters can induce significant inertia forces at small vibration amplitudes due to their 
(a)

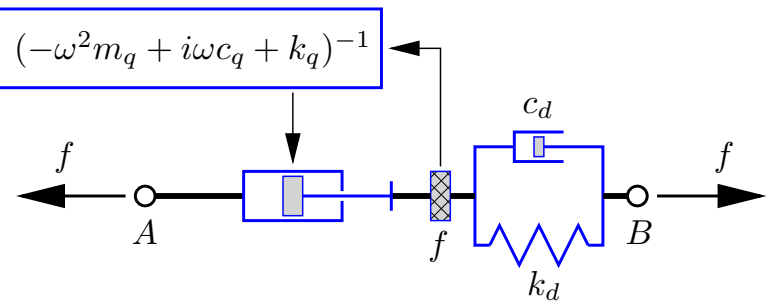

(b)

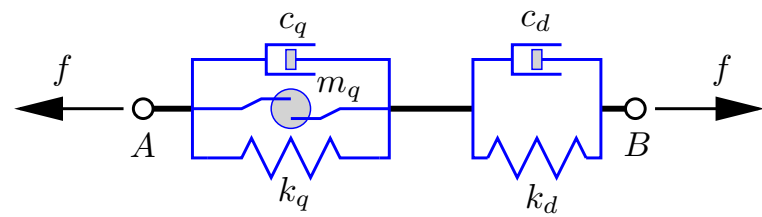

(c)

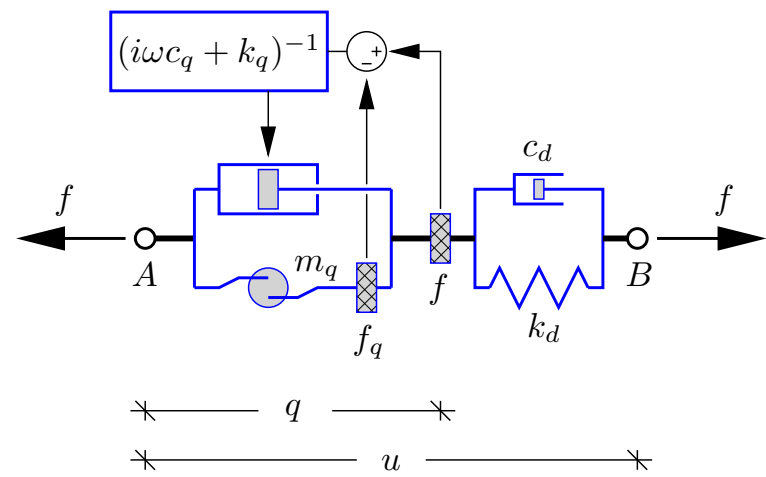

FigURE 3. Resonant passive-active vibration absorber with (a) an active hybrid unit, (b) an equivalent mechanical model and (c) a combined format with first order integration and mechanical inertia.

large gearing ratios of more than 1:200, see [4]. Furthermore, the stiffness contribution $k_{q}$ could be divided into a passive and an active part to secure a restoring force alongside the mechanical inerter component.

\section{Modal equations}

Resonant vibration damping requires accurate parameter calibration with respect to the modal properties of the targeted vibration form of the flexible structure. The mode shape vector $\mathbf{u}_{s}$ and the natural angular frequency $\omega_{s}$ of the structure are determined by the eigenvalue problem associated with (1),

$$
\left(\mathbf{K}-\omega_{s}^{2} \mathbf{M}\right) \mathbf{u}_{s}=\mathbf{0}
$$

The modal equation of motion with respect to the normalized mode shape vector $\mathbf{u}_{s} /\left(\mathbf{w}^{T} \mathbf{u}_{s}\right)$ follows from (1) as

$$
\left(-\omega^{2} m_{s}+k_{s}\right) u_{s}+f=f_{e}
$$

with modal mass $m_{s}$, modal stiffness $k_{s}$, modal load $f_{e}$ and natural frequency defined by the stiffness to mass ratio $\omega_{s}^{2}=k_{s} / m_{s}$. 
In (8) the displacement $u$ contains contributions from both the targeted vibration form and the residual non-resonant modes. As demonstrated in $[25,26]$ the influence of spill-over from nonresonant modes can be represented by the extended modal representation

$$
u=u_{s}-\frac{\kappa_{s}}{k_{s}} f
$$

where $u_{s}$ is the modal coordinate, while $\kappa_{s}$ is a non-dimensional flexibility parameter

$$
\kappa_{s}=k_{s}\left(\mathbf{w}^{T} \mathbf{K}^{-1} \mathbf{w}\right)-1
$$

representing the quasi-static influence from the non-resonant vibration modes of the structure. In (14) $k_{s}$ represents the structural stiffness over the absorber terminals when considering only the resonant mode, while $\mathbf{w}^{T} \mathbf{K}^{-1} \mathbf{w}$ is the quasi-static flexibility over the absorber terminals when including all modes. Consequently the first term in (14) is always larger than unity and $\kappa_{s}$ is therefore non-negative. The quasi-static correction in (14) is suitable for damping of the first vibration mode, while an augmented quasi-dynamic correction has recently been developed for targeting higher modes [27].

The force relation (8) is now expressed in terms of the modal displacement $u_{s}$ by substitution of (13),

$$
\frac{f}{u_{s}}=\frac{H_{d}(\omega)\left(H_{q}(\omega)+G_{q}(\omega)\right)}{H_{d}(\omega)+H_{q}(\omega)+G_{q}(\omega)++\left(\kappa_{s} / k_{s}\right) H_{d}(\omega)\left(H_{q}(\omega)+G_{q}(\omega)\right)}
$$

The combined modal equations (12) and (15) now govern the dynamics of the targeted vibration form and the corresponding characteristic equation in $\omega$ is derived from the denominator of the corresponding frequency response function $u_{s} / f_{e}$. In the following this characteristic equation will be the foundation of explicit parameter calibration based on equal modal damping.

\section{Resonant absorber CALIBRATion}

Resonant vibration absorbers are effectively calibrated using the balanced concept of equal modal damping $[26,28]$. It is a pole-placement approach where the coefficients of the quartic characteristic equation in $\omega$ are determined by comparison with a generic equation, constructed to secure equal damping of the two modes associated with the targeted vibration form. Two absorber configurations are treated in this section as they are explicitly tunable by this balanced concept.

4.1. The inertia-based absorber. The mathematical form of the mechanical tuned mass damper $[1,2]$ in Fig. 1(b) and the tuned inerter damper [6] are both recovered by $c_{q}=0$ and $k_{q}=0$ in Fig. 3, whereby only the inertia term is retained in the resulting transfer function $H_{q}(\omega)+G_{q}(\omega)=-\omega^{2} m_{q}$ in (10). This vibration absorber is therefore inertia-based, as the magnitude of $m_{q}$ determines the attainable damping. The inertance $m_{q}$ and the mechanical stiffness $k_{d}$ and damping $c_{d}$ are conveniently represented in terms of the mass ratio $\mu$, absorber frequency $\omega_{d}$ and damping ratio $\zeta_{d}$

$$
\mu=\frac{m_{q}}{m_{s}} \quad, \quad \omega_{d}^{2}=\frac{k_{d}}{m_{q}} \quad, \quad 2 \zeta_{d} \omega_{d}=\frac{c_{d}}{m_{q}}
$$


Because of the equivalence with the tuned mass damper the governing absorber parameters are directly determined by the calibration expressions presented in [25],

$$
\omega_{d}^{2}=\frac{\omega_{s}^{2}}{(1+\mu)^{2}-\mu \kappa_{s}} \quad, \quad \zeta_{d}^{2}=\frac{1}{2} \frac{\mu(1+\mu)}{(1+\mu)^{2}-\mu \kappa_{s}}
$$

The actual absorber parameters $m_{q}, k_{d}$ and $c_{d}$ are subsequently obtained by (16) for a given mass ratio $\mu$, conveniently determined with respect to a desired supplemental damping ratio $\zeta_{\text {des }}$ as

$$
\mu=\frac{8 \zeta_{\mathrm{des}}^{2}}{1-8 \zeta_{\mathrm{des}}^{2}}
$$

This explicit parameter calibration is also directly applicable for the tuned inerter damper considered in [6], with optimal absorber location being the only difference to the tuned mass damper [25]. For the pure passive absorber the inertia in $H_{q}(\omega)=-\omega^{2} m_{q}$ is realized by a mechanical inerter element, while for the active realization the control equation $G_{q}(\omega)=-\omega^{2} m_{q}$ contains a double integration of the measured absorber force, which might be sensible to actuator saturation from low-frequency sensor input [29].

4.2. The stiffness-based absorber. An alternative absorber configuration is now proposed, corresponding to the resonant vibration absorber in Fig. 3 without passive damping $\left(c_{d}=0\right)$ and consequently with $H_{d}(\omega)=k_{d}$. This vibration absorber is therefore stiffness-based because the amount of attainable damping is governed by the stiffness $k_{d}$ of the passive spring. The stiffness $k_{q}$ in the hybrid unit is specifically introduced to avoid actuator saturation associated with an active force feedback realization. The stiffness-based absorber is therefore introduced as a fourparameter absorber. In active vibration control $k_{d}$ could represent the mechanical stiffness of a piezoelectric transducer [29], with elongation $q$ controlled by the applied voltage using the filtered force feedback equation (5) with $G_{q}(\omega)=-m_{q} \omega^{2}+i \omega c_{q}+k_{q}$ and $f_{q}=0$.

The calibration of the stiffness-based absorber follows the equal modal damping procedure outlined in [28] for a family of resonant control formats and in [26] for piezoelectric damping with $R L$ (resistive-inductive) shunt circuits. The characteristic equation is obtained from the denominator of the frequency response function $u_{s} / f_{e}$,

$$
\left(-\omega^{2}+\omega_{s}^{2}\right)\left(-\omega^{2}+2 i \zeta_{q} \omega_{d} \omega+(1+\alpha) \omega_{d}^{2}\right)+\nu\left(-\omega^{2}+2 i \zeta_{q} \omega_{d} \omega+\alpha \omega_{d}^{2}\right)\left(\omega_{s}^{2}\right)\left[1+\kappa_{s}\left(1-\frac{\omega^{2}}{\omega_{s}^{2}}\right)\right]=0
$$

introducing the stiffness ratios $\nu$ and $\alpha$ and the filter damping ratio $\zeta_{q}$ as

$$
\nu=\frac{k_{d}}{k_{s}} \quad, \quad \alpha=\frac{k_{q}}{k_{d}} \quad, \quad 2 \zeta_{q} \omega_{d}=\frac{c_{q}}{m_{q}}
$$

with $\omega_{d}$ given in (16b). For $\alpha=0$ the equation (19) exactly recovers the characteristic equation for piezoelectric damping with a series $R L$ shunt circuit. Therefore, the calibration procedure in [26] is conveniently applied with the two stiffness ratios $\nu$ and $\alpha$ as given system parameters, and with $\omega_{d}$ and $\zeta_{q}$ determined by the calibration procedure,

$$
\omega_{d}^{2}=\frac{\left(1+\left(1+\kappa_{s}\right) \nu\right)^{2}}{\left(1+\nu \kappa_{s}\right)\left[1+\alpha\left(1+\nu\left(1+\kappa_{s}\right)\right)\right]} \quad, \quad \zeta_{q}^{2}=\frac{1}{2} \frac{\nu}{\left(1+\nu \kappa_{s}\right)\left(1+\left(1+\kappa_{s}\right) \nu\right)}
$$

The actual absorber stiffness components are determined as $k_{d}=\nu k_{s}$ and $k_{q}=\alpha k_{d}$ for given values of $\nu$ and $\alpha$, respectively. Subsequently, the inertance $m_{q}$ and damping $c_{q}$ are obtained 
from (21) together with (16b) and (20c), respectively. For the present stiffness-based absorber the stiffness ratio $\nu$ is chosen with respect to a desired level of supplemental damping $\zeta_{\text {des }}$. According to [26] the stiffness ratio $\nu$ should therefore be determined as

$$
\nu=\frac{8 \zeta_{\text {des }}^{2}(1+\alpha)}{1-8 \zeta_{\text {des }}^{2}\left[\kappa_{s}+\alpha\left(1+2 \kappa_{s}\right)\right]}
$$

assuming that the stiffness ratio $\alpha$ of the hybrid unit has been chosen to secure suitable filter properties in the control equation (5).

\section{DAmping of FleXible Structure}

Figure 4 shows a cantilever beam of length $\ell=20 a$, discretized into 20 Bernoulli beam elements of length $a$ and with transverse displacement $x_{j}$ and rotation $\varphi_{j}$ as the nodal degrees of freedom. A pair of passive-active vibration absorbers are placed, symmetrically with respect to the neutral beam axis, at distance $\ell_{a}$ from the support. The absorber pair acts on the nodal rotations of a single beam element, whereby the connectivity vector is represented as

$$
\mathbf{w}=[\ldots 0, \underbrace{0-1,0,1}_{\text {absorber element }}, 0 \ldots]^{T}
$$

Two different absorber locations with $\ell_{a}=\ell / 4=5 a$ and $\ell_{a}=\ell / 2=10 a$ are considered to investigate the performance with respect to limited authority. The normalized modal mass $m_{s}$ is 3.5 times larger for $\ell_{a}=\ell / 2$ compared to $\ell_{a}=\ell / 4$, which demonstrates that the attainable damping at the center position is reduced significantly compared to the location closer to the support. The background flexibility parameter in (14) also increases from $\kappa_{s}=9.45$ at $\ell_{a}=\ell / 4$ to $\kappa_{s}=35.71$ for $\ell_{a}=\ell / 2$. In both cases $\kappa_{s} \gg 1$ and effective damping therefore requires the inclusion of $\kappa_{s}$ in the absorber tuning.

5.1. Complex frequency analysis. The closed-loop equations are comprised by (1) and (8), and the free vibration properties are therefore determined by solving the eigenvalue problem associated with $\mathbf{f}_{e}=\mathbf{0}$. Figure 5 shows the trajectories of the two complex-valued natural frequencies $\omega$ associated with the first vibration form of the beam structure. Damping is conveniently assessed in terms of the damping ratio $\zeta=\operatorname{Im}[\omega] /|\omega|$, which for a single mode system defines the resonant dynamic amplification as $1 /(2 \zeta)$. The pair of blue solid curves $(+, \times)$ represent the inertia-based vibration absorber with $H_{d}(\omega)=i \omega c_{d}+k_{d}$ and $H_{q}(\omega)+G_{q}(\omega)=-\omega^{2} m_{q}$, while the two pairs

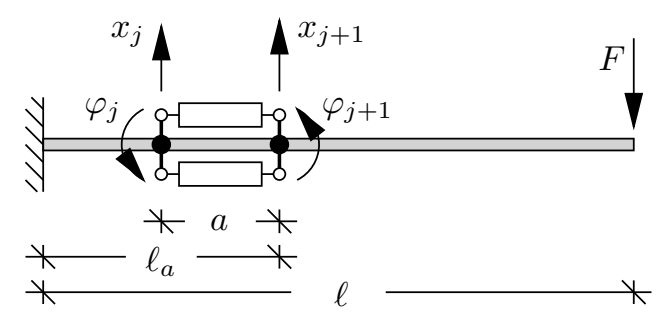

FIGURE 4. Cantilever beam with passive-active absorber pair. 
(a)

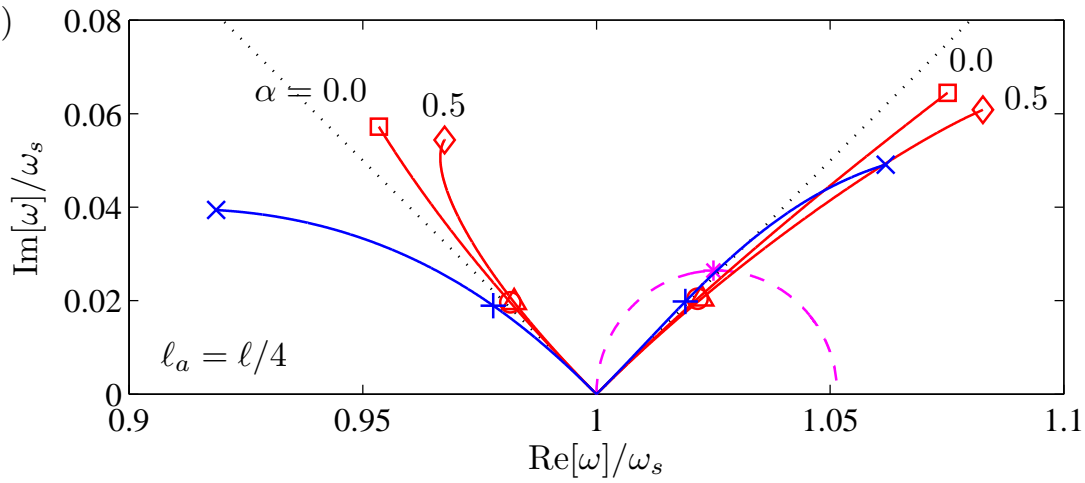

(b)

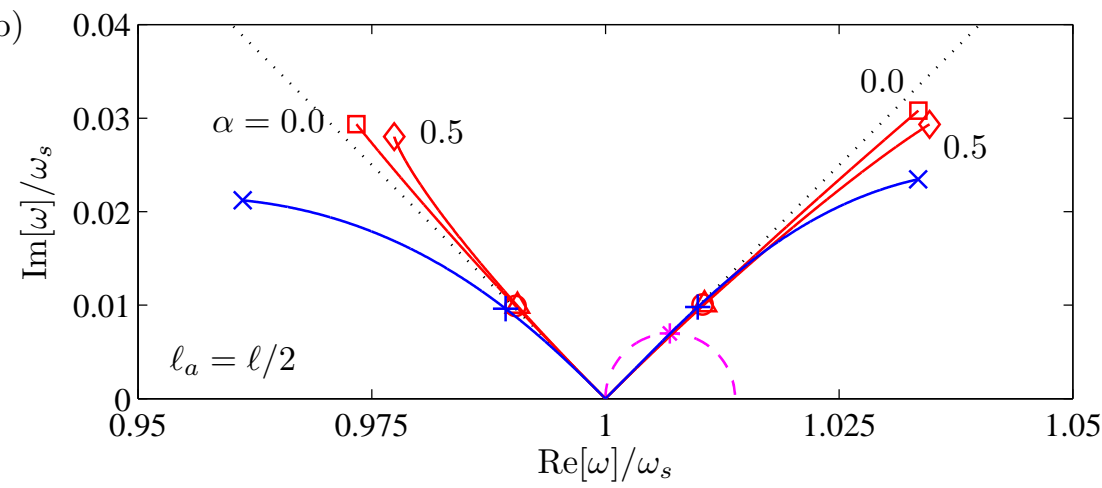

Figure 5. Trajectory of complex natural frequencies: (a) $\ell_{a}=\ell / 4$ with $\zeta_{\text {des }}=$ $0.02(+, \bigcirc, \triangle)$ and $0.06(\times, \square, \diamond),(\mathrm{b}) \ell_{a}=\ell / 2$ with $\zeta_{\text {des }}=0.01(+, \bigcirc, \triangle)$ and 0.03 $(\times, \square, \diamond)$. Optimal viscous damping $(*)$.

of red solid curves represent the stiffness-based absorber with $H_{d}(\omega)=k_{d}$ and $H_{q}(\omega)+G_{q}(\omega)=$ $-\omega^{2} m_{q}+i \omega c_{q}+k_{q}$ and with stiffness ratio $\alpha=0.0(\bigcirc, \square)$ and $0.5(\triangle, \diamond)$. The dashed magenta curve represents the benchmark trajectory of a pure viscous damper $\left(c_{d}\right)$ obtained by $q=0$ and $k_{d}=0$. The trajectory for the pure viscous damper is approximately semicircular, as discussed in [30]. The figures 5(a) and (b) represent the two damper locations $\ell_{a}=\ell / 4$ and $\ell / 2$, respectively. The markers identify the natural frequencies obtained for the desired damping ratios $\zeta_{\text {des }}=0.02(+, \bigcirc, \triangle)$ and $0.06(\times, \square, \diamond)$ for $\ell_{a}=\ell / 4$, and $\zeta_{\text {des }}=0.01(+, \bigcirc, \triangle)$ and $0.03(\times, \square, \diamond)$ for $\ell_{a}=\ell / 2$. The calibration results for the inertia-based absorber are summarized in Table 1, while the corresponding results for stiffness-based configuration are presented in Table 2. In both tables the two final columns provide the damping ratios $\zeta$ for the two modes associated with the first vibration form of the structure.

The equal modal damping calibration of resonant vibration absorbers implies that the complex roots lie on the same line through the origin of the complex plane, while the desired damping ratio $\zeta=\zeta_{\text {des }}$ corresponds to trajectories along $\pm 45^{\circ}$ paths, indicated by the dotted lines in Fig. 5 . In depth explanations of the equal modal damping properties are provided in [28]. It is seen in Fig. 5 that the trajectories of stiffness-based absorber follow the desired $\pm 45^{\circ}$ lines more accurately than for the inertia-based absorber, where in particular the low-frequency branch deviates from the 
TABLE 1. Parameters and damping ratios for inertia-based absorber $\left(c_{q}=0, k_{q}=0\right)$.

\begin{tabular}{cccccccc}
\hline$\ell_{a}$ & $\zeta_{\mathrm{des}}$ & $\mu$ & $\frac{\omega_{d}}{\omega_{s}}$ & $\zeta_{d}$ & \multicolumn{2}{c}{$\zeta$} & \\
\hline \multirow{2}{*}{$\ell / 4$} & 0.020 & 0.0032 & 1.0122 & 0.0406 & 0.0194 & 0.0195 & $(+)$ \\
& 0.060 & 0.0297 & 1.1324 & 0.1399 & 0.0430 & 0.0464 & $(\times)$ \\
\hline \multirow{2}{*}{$\ell / 2$} & 0.010 & 0.0008 & 1.0138 & 0.0203 & 0.0097 & 0.0097 & $(+)$ \\
& 0.030 & 0.0073 & 1.1504 & 0.0695 & 0.0221 & 0.0227 & $(\times)$ \\
\hline
\end{tabular}

desired path. As seen in Table 1 the inertia-based absorber leads to almost equal modal damping, however slightly below $\zeta_{\text {des }}$, while for stiffness-based configuration in Table $2 \zeta_{\text {des }}$ is accurately reproduced. For the optimal viscous damping represented by the magenta asterisks the maximum damping ratios are $\zeta_{\text {vis }}=0.0258$ for $\ell_{a}=\ell / 4$ and 0.0069 for $\ell_{a}=\ell / 2$. Thus, compared to optimal viscous damping both vibration absorber configurations yield a substantial increase in damping ratio by a factor of two for $\ell_{a}=\ell / 4$ and four for the more indirect location at $\ell_{a}=\ell / 2$.

5.2. Frequency response analysis. The frequency response characteristics are investigated by applying a harmonic tip load with frequency amplitude $F$ in $\mathbf{f}_{e}$. The dynamic amplification factor of the transverse tip displacement $u_{\text {tip }}$ is shown in Fig. 6(a,b), while the curves in Fig. 6(c,d) present the frequency amplitude of the resulting absorber force $f$. The left and right pair of figures represent the absorber locations $\ell_{a}=\ell / 4$ and $\ell / 2$, respectively. The two blue curves show the inertia-based absorber, while the four red curves represent stiffness-based type. The individual markers identify the individual curves with respect to the parameter calibration and results presented in Fig. 5 and Tables 1 and 2. Finally, the dashed magenta curve represents the optimal viscous damper associated with the asterisks in Fig. 5.

For $\ell_{a}=\ell / 4$ in Fig. 6 (a) the vibration amplitudes at resonance correspond well with the singlemode estimate $1 /\left(2 \zeta_{\text {des }}\right)$. For $\zeta_{\text {des }}=0.02(+, O, \triangle)$ the response curves are very similar for the two absorber configurations, while for $\zeta_{\text {des }}=0.06(\times, \square, \diamond)$ the stiffness-based absorber produces

TABLE 2. Parameters and damping ratios for stiffness-based absorber $\left(c_{d}=0\right)$.

\begin{tabular}{ccccccccc}
\hline$\ell_{a}$ & $\zeta_{\text {des }}$ & $\alpha$ & $\mu$ & $\frac{\omega_{d}}{\omega_{s}}$ & $\zeta_{q}$ & \multicolumn{2}{c}{$\zeta$} & \\
\hline \multirow{4}{*}{$\ell / 4$} & 0.020 & 0.0 & 0.0033 & 1.0187 & 0.0393 & 0.0200 & 0.0200 & $(\bigcirc)$ \\
& 0.060 & 0.0 & 0.0396 & 1.2060 & 0.1009 & 0.0599 & 0.0599 & $(\square)$ \\
& 0.020 & 0.5 & 0.0051 & 0.8327 & 0.0481 & 0.0200 & 0.0200 & $(\triangle)$ \\
& 0.060 & 0.5 & 0.0980 & 1.0282 & 0.1121 & 0.0561 & 0.0561 & $(\diamond)$ \\
\hline \multirow{4}{*}{$\ell / 2$} & 0.010 & 0.0 & 0.0008 & 1.0154 & 0.0197 & 0.0100 & 0.0100 & $(\bigcirc)$ \\
& 0.030 & 0.0 & 0.0097 & 1.1685 & 0.0515 & 0.0302 & 0.0298 & $(\square)$ \\
& 0.030 & 0.5 & 0.0224 & 0.9825 & 0.0584 & 0.0287 & 0.0284 & $(\diamond)$ \\
\hline
\end{tabular}



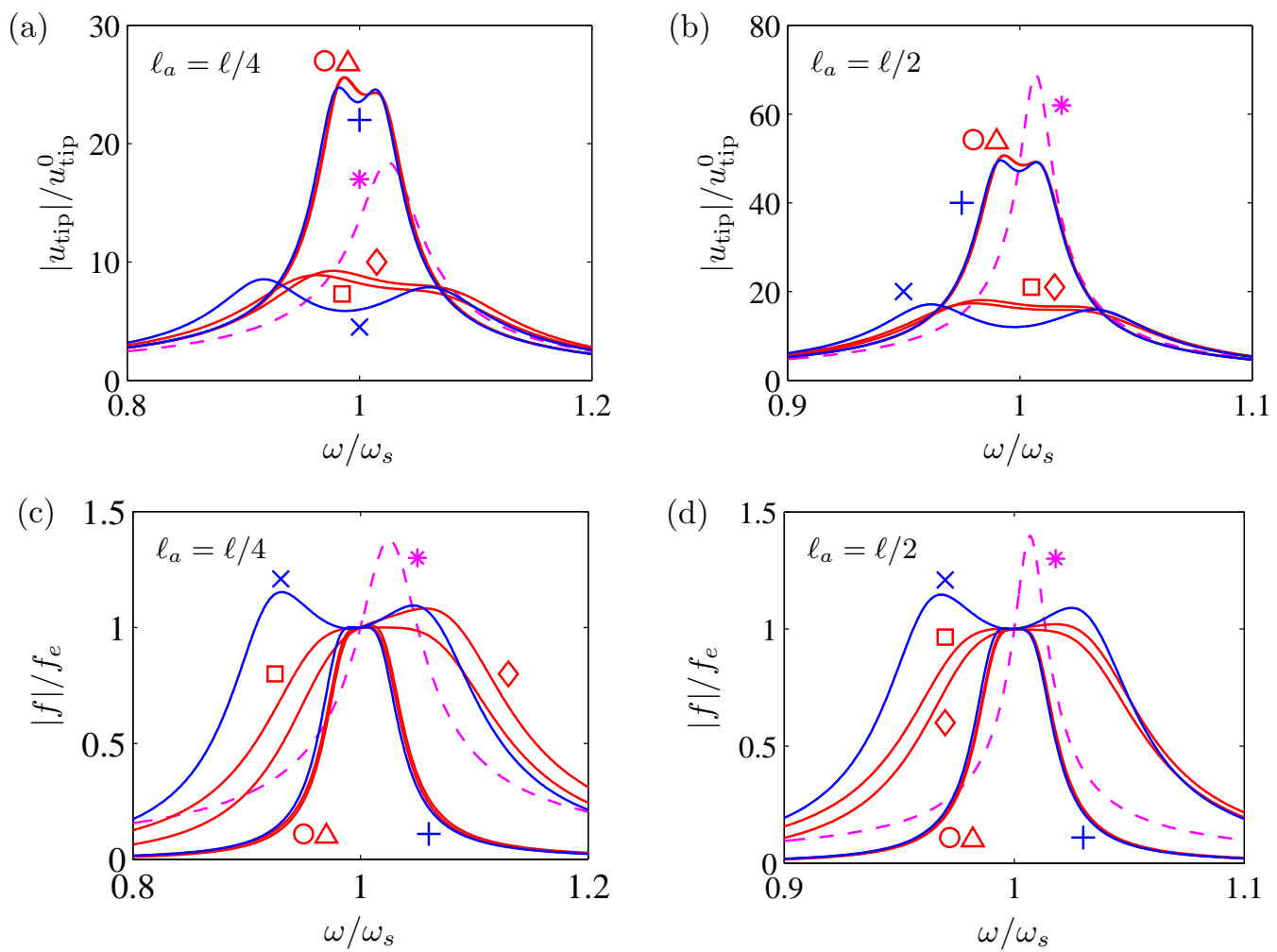

Figure 6. Frequency response amplitudes for $\ell_{a}=\ell / 4(\mathrm{a}, \mathrm{c})$ with $\zeta_{\mathrm{des}}=0.02$ $(+, \bigcirc, \triangle)$ and $0.06(\times, \square, \diamond)$ and for $\ell_{a}=\ell / 2(\mathrm{~b}, \mathrm{~d})$ with $\zeta_{\mathrm{des}}=0.01(+, \bigcirc, \triangle)$ and $0.03(\times, \square, \diamond)$. Optimal viscous damping $(*)$.

a slightly more flat plateau. The frequency amplitude of the absorber force in Fig. 6(c) is ideally flat for $\zeta_{\text {des }}=0.02(+, \bigcirc, \triangle)$, while local double peaks appear for the inertia-based absorber with $\zeta_{\text {des }}=0.06(\times)$. In the stiffness-based configuration the non-vanishing filter stiffness ratio $\alpha=0.5(\diamond)$ only introduces a minor deterioration of the absorber performance, which suggests that $k_{q}$ should be used in the active control function $G_{q}(\omega)$ to secure a proper integration in (5). The magnitude of the viscous damper force in Figs. $6(\mathrm{c}, \mathrm{d})$ is larger than the corresponding absorber force, which demonstrates that the proposed passive-active vibration absorber achieves a significant response reduction at lower force levels compared to the viscous damper. In particular, the results in Figs. 6(b,d) for $\ell_{a}=\ell / 2$ show that compared to the optimal viscous damper the passive-active vibration absorber is very effective at indirect absorber locations, provided that the $\kappa_{s}$-correction is included in the calibration formulae.

\section{Summary}

A general passive-active absorber format contains an integrated force feedback control with sensor input represented by the difference in force between the two passive absorber elements. A five-parameter resonant vibration absorber is investigated in detail, where $k_{d}$ and $c_{d}$ constitute passive stiffness and damping, while the resulting inertance $m_{q}$, damping $c_{q}$ and stiffness $k_{q}$ of the 
hybrid unit can be realized by a combination of passive and active contributions due to the format of the proposed force feedback control. Two particular configurations are presented because they are tunable by the equal modal damping procedure described in $[26,28]$. For both configurations explicit design expressions are presented for the absorber parameters, which take into account the influence from residual vibration modes by the background flexibility parameter $\kappa_{s}$. The results show that the proposed passive-active vibration absorber introduces large attainable damping and provides effective resonant response reduction with limited absorber force amplitudes. In particular, the stiffness-based absorber demonstrates good damping performance and is furthermore suitable for active realization due to the non-vanishing $k_{q}$ and $c_{q}$ components in $G_{q}(\omega)$, which limits the influence from actuator saturation. The specific absorber configuration of course depends on the particular problem, and obviously the robustness of pure passive absorbers is attractive. However, the vibration absorber is apparently effective at very indirect absorber locations with limited authority and absorber displacement. Thus, it might be applicable for smart actuators of for example piezoelectric type, in which $k_{d}$ and $c_{d}$ then represent mechanical stiffness and hysteresis, respectively, while the elongation $q$ is actively controlled via the applied voltage across the transducer electrodes [29].

\section{REFERENCES}

[1] Den Hartog JP (1956) Mechanical Vibrations 4th edition. New York: McGraw-Hill.

[2] Krenk S (2005) Frequency analysis of the tuned mass damper. Journal of Applied Mechanics 72: 936-942.

[3] Smith MC (2002) Synthesis of mechanical networks: The inerter. IEEE/ASME Transactions on Automatic Control 47: 1648-1662.

[4] Chen MZQ, Papageorgiou C, Scheibe F, Wang FC and Smith MC (2009) The missing mechanical circuit element. IEEE Circuits and systems Magazine 9: 10-26.

[5] Ikago K, Saito K and Inoue N (2012) Seismic control of single-degree-of-freedom structure using tuned viscous mass damper. Earthquake Engineering and Structural Dynamics 41: 453474.

[6] Lazar IF, Neild SA and Wagg DJ (2014) Using an inerter-based device for structural vibration suppression. Earthquake Engineering and Structural Dynamics 43: 1129-1147.

[7] Marian L and Giaralis A (2014) Optimal design of a novel tuned mass-damper-inerter (TMDI) passive vibration control configuration for stochastically support-excited structural systems. Probabilistic Engineering Mechanics 38: 156-164.

[8] Hu Y, Chen MZQ, Shu Z and Huang L (2015) Analysis and optimisation for inerter-based isolators via fixed-point theory and algebraic solution. Journal of Sound and Vibration 346: 1736.

[9] Caruso G (2001) A critical analysis of electric shunt circuits employed in piezoelectric passive vibration damping. Smart Materials and Structures 10: 1059-1068. 
[10] Soltani P, Kerschen G, Tondreau G and Deraemaeker A (2014) Piezoelectric vibration damping using resonant shunt circuits: an exact solution. Smart Materials and Structures 23: 125014 (11pp).

[11] Behrens S, Fleming AJ and Moheimani SOR (2005) Passive vibration control via electromagnetic shunt damping. IEEE/ASME Transactions on Mechatronics 10: 118-122.

[12] Zhu S, Shen W and Qian X (2013) Dynamic analogy between an electromagnetic shunt damper and a tuned mass damper. Smart Materials and Structures 22: 115018 (11pp).

[13] Zuo Land Cui W (2013) Dual-functional energy-harvesting and vibration control: electromagnetic resonant shunt series tuned mass dampers. Journal of Vibration and Acoustics 135: 051018 (9pp).

[14] Gonzalez-Buelga A, Clare LR, Neild SA, Jiang JZ and Inman DJ (2015) An electromagnetic inerter-based vibration suppression device. Smart Materials and Structures 24: 055015 (10pp).

[15] Lee-Glauser G, Juang JN and Sulla JL (1995) Optimal active vibration absorber: Design and experimental results. Journal of Vibration and Acoustics 117: 165-171.

[16] Juang JN and Phan M (1992) Robust controller designs for second-order dynamic systems: A virtual passive approach. Journal of Guidance, Control, and Dynamics 15: 1192-1198.

[17] Wu ST, Shao YJ (2007) Adaptive vibration control using a virtual-vibration-absorber controller. Journal of Vibration and Control 305: 891-903.

[18] de Noyer MPB and Hanagud SV (1998) Robust controller designs for second-order dynamic systems: A virtual passive approach. Journal of Intelligent Material Systems and Structures 9: $522-533$.

[19] Krenk S and Høgsberg J (2009) Optimal resonant control of flexible structures. Journal of Sound and Vibration 323: 530-554.

[20] Preumont A, Dufour J-P and Malékian C (1992) Active damping by a local force feedback with piezoelectric actuators. Journal of Guidance, Control, and Dynamics 15: 390-395.

[21] Preumont A and Achkire Y (1997) Active damping of structures with guy cables. Journal of Guidance, Control, and Dynamics 20: 320-326.

[22] Preumont A, Francois A, Bossens F and Abu-Hanieh A (2002) Force feedback versus acceleration feedback in active vibration isolation. Journal of Sound and Vibration 257: 605-613.

[23] Teo YR and Fleming AJ (2015) Optimal integral force feedback for active vibration control. Journal of Sound and Vibration 356: 20-33.

[24] Høgsberg J and Brodersen ML (2015) Hybrid viscous damper with filtered integral force feedback control. Journal of Vibration and Control DOI: 10.1177/1077546314543912.

[25] Krenk S and Høgsberg J (2014) Tuned mass absorber on a flexible structure. Journal of Sound and Vibration 333: 1577-1595.

[26] Høgsberg J and Krenk S (2015) Balanced calibration of resonant piezoelectric RL shunts with quasi-static background flexibility correction. Journal of Sound and Vibration 341: 16-30.

[27] Krenk S and Høgsberg J (2015) Tuned resonant mass or inerter based absorbers: Unified calibration with quasi-dynamic flexibility and inertia correction. submitted for publication. 
[28] Krenk S and Høgsberg J (2013) Equal modal damping design for a family of resonant vibration control formats. Journal of Vibration and Control 19: 1294-1315.

[29] Preumont A (2011) Vibration Control of Active Structures. An Introduction, 3rd edition. Springer, Heidelberg.

[30] Main JA and Krenk S (2005) Efficiency and tuning of viscous dampers on discrete systems. Journal of Sound and Vibration 286: 97-122. 